ferritin and d-dimer levels, with high C3, IL-6 and TNF-alpha levels. Microbial cultures (blood cultures, nasopharyngeal and throat swab) were sterile, and serology for Cytomegalovirus, Epstein-Barr virus, Human parvovirus B19 and Bartonella henselae were negative, while serology for Mycoplasma pneumoniae (IgM and IgG) was positive. Azithromycin was prescribed, but fever and elevated inflammatory blood markers persisted and other procedures were performed to rule out rheumatic disease. Radiogram and bone scintigraphy were unremarkable, but an ultrasound revealed a retroperitoneal mass. MRI was performed, and retroperitoneal mass measuring $3 \times 2.5 \mathrm{~cm}$, arising from the pancreas and suspected to be a neuroendocrine tumor or enlarged lymph node, along with hepatosplenomegaly was described.

FDG-positron emission tomography (PET)/CT showed a mass near the pancreas with moderately increased FDG uptake. Complete surgical resection of the mass was performed, and the histopathological evaluation revealed lymphoid hyperplasia consistent with the hyaline vascular type of Castleman disease.

An immunohistochemical study excluded HHV-8 positivity. After the surgical procedure, the patient recovered completely. On follow up visits he was without fever or other symptoms suggestive of the disease, with normal laboratory findings.

$\mathrm{CD}$ with systemic manifestations is rare in children emphasizing the need for thorough evaluation of unexplained prolonged symptoms.

\section{SEVERE SKIN MANIFESTATIONS IN PATIENTS WITH HENOCH SCHOENLEIN PURPURA (HSP) IN FIVE TERTIARY CENTRES IN CROATIA BETWEEN 2009 AND 2019}

Saša Sršen*, Mateja Batnožić Varga, Matej Šapina, Mario Šestan, Nastasia Čekada, Aleksandar Ovuka, Marijan Frković, Alenka Gagro, Marija Jelušić. University Hospital Centre Split, University of Split School of Medicine

10.1136/archdischild-2021-europaediatrics.435

To evaluate characteristics of patients with severe skin manifestations of Henoch Schoenlein purpura (HSP) in five tertiary centres in Croatia in time period from 2009 until 2019.

Retrospective statistical chart analysis of clinical symptoms and laboratory parameters of patients with HSP was done. Severe skin manifestations were defined as haemorrhagic bullous, ulcerated or necrotic lesions.

During observed time period 583 patients $(303$ boys and 280 girls) were admitted to hospital due to HSP. Prevalence of severe skin manifestations was $2.41 \%$ (14 patients, CI 1.32 - 4.003\%). Severe skin manifestations occurred more often in boys than in girls with a difference on a margin of statistical significance ( 11 boys, 3 girls, $\mathrm{p}=0.057)$. Patients with severe skin manifestations were more prone to relapses than those with milder skin forms (severe 28.57\%, milder 18.66\%), with most notable difference in patients with 2 relapses of disease (severe 14.29\%, milder 3.17\%, $\mathrm{p}=0.029$ ).

We were able to isolate infectious trigger of disease more often in patients with milder form of disease than in those with more severe (milder 38.42\%, severe $7.69 \%, \mathrm{p}=0.036$ ).

Patients with severe skin manifestations had more often gastrointestinal system affected than those with milder skin forms (severe $70 \%$, milder $36.03 \%, \mathrm{p}=0.043$ ). There was no statistically significant difference between those two groups in other organs involvement (kidneys, joints, central nervous system, lung).

Regarding distribution of skin rash, patients with severe skin manifestations more often had a rash on a trunk, with a difference on a margin of statistical significance (severe 85.71\%, milder 57.19\%, $\mathrm{p}=0.051)$.

There was no statistical difference between two groups in most of other clinical and laboratory parameters. Only blood leukocyte levels were near to statistical significance with higher levels in patients with severe skin manifestations (12.1*109/L (SD 10-17.62):10.71*109/L (SD 8.45-13.42), $\mathrm{p}=0.059)$.

Patients with severe skin manifestations of HSP were more prone to relapses, and had gastrointestinal symptoms more often affected than those with milder forms, but less often had infectious trigger isolated. There was no significant statistical difference in other clinical symptoms and laboratory parameters. We couldn't set predictive model for disease outcome based on these data. Small number of patients with severe manifestations and retrospective data analysis could be the reason. Perhaps better structured prospective trial would be more appropriate in that sense.

SUPPORT: Croatian Science Foundation project IP-2019-048822

\section{DISLIPIDEMIA AS AN ATHEROGENIC FACTOR IN PATIENTS WITH DIFFERENT FORMS OF JUVENILE ARTHRITIS}

AV Krasnopolskaya*, LA Balykova, NV Shekina, UO Soldatov, SE Pomerantceva. Medicine Institute, National Research Ogarev Mordovia State University

\subsection{6/archdischild-2021-europaediatrics.436}

Close connection between autoimmune inflammation and proatherogenic lipid changes in rheumatoid arthritis has been well established, while for juvenile idiopathic arthritis (JIA) is under discussion. The aim of our work was to study the incidence and intensity of lipid disturbances in patients with different forms of JIA.

90 children with JIA 6-18 years were examined using clinical, biochemical methods, ultrasonic duplex scanning of vessels, thin-layer chromatography, bioimpedance measurement. 49 children without chronic diseases made up the control group.

Dyslipidaemia, was revealed in $48.9 \%$ of patients, mainly with systemic and polyarthritis Most often an increase in atherogenic coefficient (AC) was noted. Average AC value in JIA patients was higher than in control group $(2.9+0.2$ versus $2.0+0.1, \mathrm{p}<0.05)$ and correlated with the disease activity index according to JADAS71 $(\mathrm{r}=0.78)$, a doctor`s global assessment of the disease severity according to VAS ( $\mathrm{r}=$ $0.61)$, the degree of joints dysfunction $(r=0.55)$, C-reactive protein $(\mathrm{r}=0.53)$ and ESR $(\mathrm{r}=0.68)$ level. An increase in the concentration of total cholesterol was observed in 28 (31.1\%) children with JIA, commonly with a long-lasting disease, and in case of CS intake. In some JIA patients atherogenic changes were detected due to apolipoprotein A1 (ApoA1) deficiency (26.7\%). Patients with JIA (maximum with 